\title{
Status of BELLE
}

\section{Shiro Suzuki}

Dept. of Physics, Nagoya University

Furo-cho, Chikusa-ku, Nagoya 464-8602, Japan

ABSTRACT: The status of BELLE/KEKB is reviewed. BELLE started data taking runs in June 1999, and collected a data sample with an integrated luminosity of $\sim 25 \mathrm{pb}^{-1}$ before the summer shutdown. Various pilot physics signals are presented to demonstrate the performance of the BELLE detector.

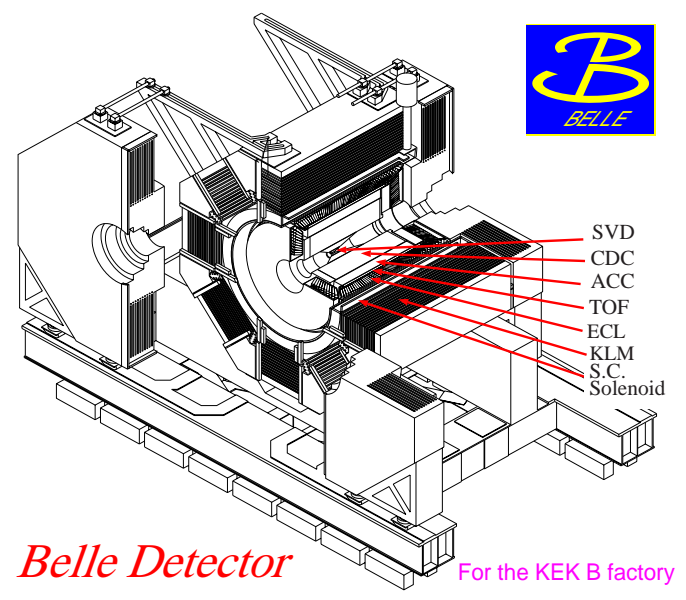

Figure 1: Schematic view of the BELLE detector.

\section{BELLE and KEKB}

The principal goal of the BELLE/KEKB is to observe $\mathrm{CP}$ violation in the $\mathrm{B}$-meson decay processes and explore its origin. BELLE is an international collaboration of 300 physicists from

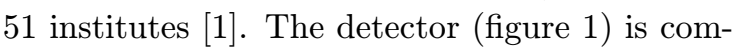
posed of a Silicon Vertex Detector (SVD), a Central Drift Chamber (CDC), Time of Flight counters (TOF), Aerogel Cherenkov Counters (ACC), an Electromagnetic Calorimeter (ECL), and a KLong / Muon catcher (KLM). A solenoid magnet provides a magnetic field of $1.5 \mathrm{~T}$ for charged particle tracking. Detailed description will be found in ref-[2].

KEKB is a $e^{+} e^{-}$collider with asymmetric collision energy of $3.5 \mathrm{GeV}\left(e^{+}\right) \times 8 \mathrm{GeV}\left(e^{-}\right)$. The beam collision angle is set to be $\pm 11 \mathrm{mrad}$

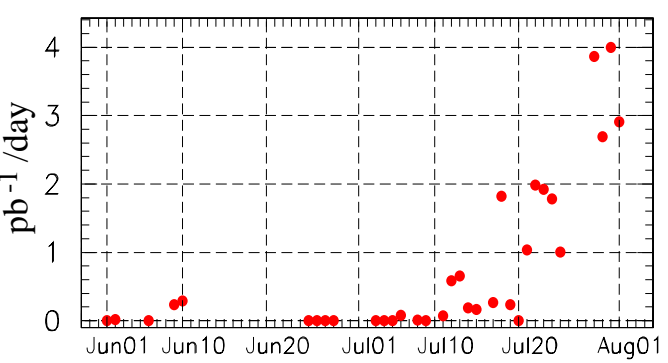

Figure 2: Progress of accumulated luminosity per day.

to avoid parasitic collisions. The design luminosity is $10^{34} \mathrm{~cm}^{-2} \mathrm{~s}^{-1}$ with 5000 bunches for both $e^{+}$and $e^{-}$. The design parameters and the parameters that have been achieved as of July 1999 are summarized in table $\underline{1}_{\mathbf{r}}$ The quoted numbers here are the best values achieved for each item and those are not necessarily consistent with each other.

\begin{tabular}{|c|c|c|}
\hline Design/achieved & $\operatorname{LER}\left(e^{+}\right)$ & $\operatorname{HER}\left(e^{-}\right)$ \\
\hline Energy $(\mathrm{GeV})$ & $3.5 / 4.0$ & $8.0 / 8.5$ \\
\hline Circumference & \multicolumn{2}{|c|}{$3016.26 \mathrm{~m}$} \\
\hline Crossing angle & \multicolumn{2}{|c|}{ $\pm 11 \mathrm{mrad}$} \\
\hline$\sigma_{x}(\mu \mathrm{m})$ & $77 / 170$ & $77 / 170$ \\
$\sigma_{y}(\mu \mathrm{m})$ & $1.9 / 3.8$ & $1.9 / 3.9$ \\
\hline No. of bunches & $5000 / 1024$ & $5000 / 800$ \\
\hline Current $(\mathrm{mA})$ & $2600 / 542$ & $1100 / 514$ \\
\hline Luminosity & \multicolumn{2}{|c|}{$1 \times 10^{34} / 1.2 \times 10^{32}$} \\
\hline
\end{tabular}

Table 1: Design / achieved parameters of KEKB

KEKB started its commissioning in December 1998 with BELLE at the roll out position. 
BELLE rolled onto beam line on May 1, 1999, and we recorded the first collision in early June. We started with the average luminosity of $2 \times$ $10^{31} \mathrm{~cm}^{-2} \mathrm{~s}^{-1}$ and reached an averaged luminosity of $1.2 \times 10^{32} \mathrm{~cm}^{-2} \mathrm{~s}^{-1}$ with beam currents of $200 \mathrm{~mA}$ (LER) and $100 \mathrm{~mA}$ (HER) by the middle of July. The plot (figure of daily accumulated luminosity. After completing an energy scan to confirm the $\Upsilon(4 S)$ peak, we carried out "luminosity runs". By the summer shutdown we reached an integrated lumnosity of $\sim 25 p b^{-1}$ and have accumulated about $60 \mathrm{~K}$ hadronic events.

In this report, the performance of the BELLE detector and some of the physics signals will be presented. Note that the results shown here are preliminary, mostly from the data sample by the middle of July, and calibration for some subdetectors are still in progress.

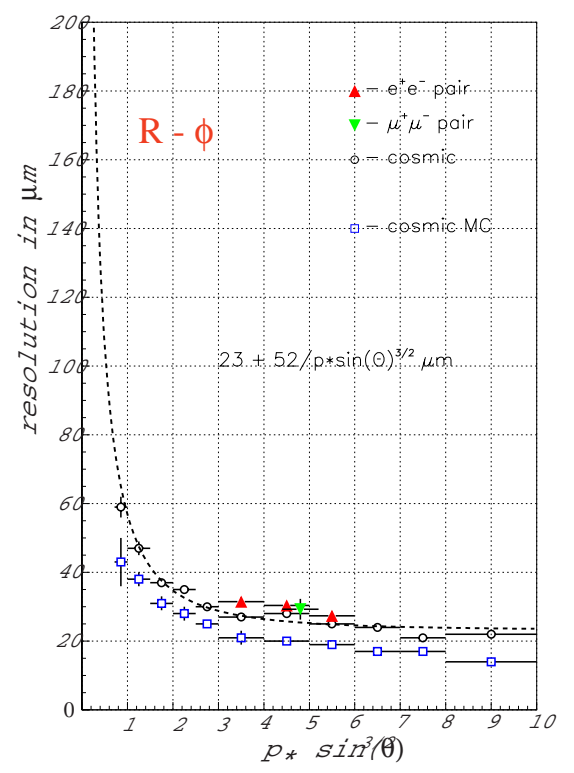

Figure 3: SVD vertex resolution in $R \phi$ as a function of $p \cdot \sin (\theta)^{3 / 2}$.

\section{Vertexing and tracking}

The SVD has a simple cylindrical structure with 3 layers of DSSD, placed at a radial distance of $3.0-5.8 \mathrm{~cm}$ from the beam axis. Two track mismatch of back to back events (like Bhabhas or $\mu$-pairs) is a good measure of $r \phi$ vertex resolution. With Bhahhas we obtain a resolution of about $35 \mu \mathrm{m}$. Data has been collected in wider $p$ and $\theta$ range using cosmic ray events (figure $\overline{3_{1}^{1}}$ ). Beam collision results of Bhabhas and $\mu$-pairs are plotted in the same figure. In the $z$ direction only cosmic ray calibration has been possible so far.

$\Delta$ Pt for cosmic ray tracks

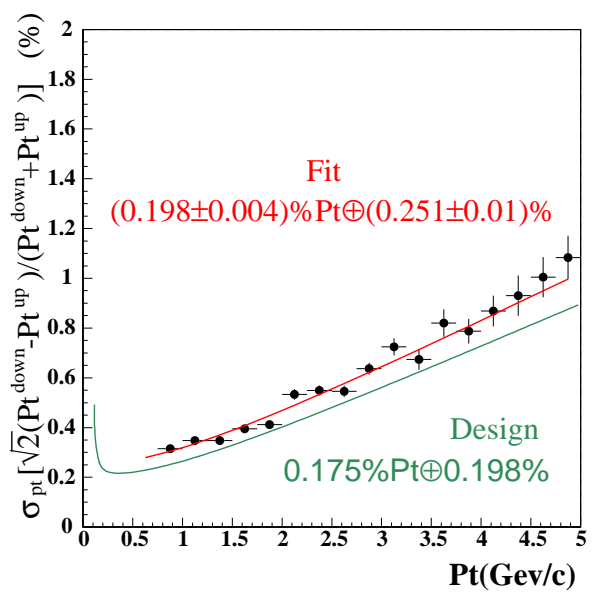

Figure 4: CDC resolution by cosmic rays.

\section{$K_{S}$ reconstruction}

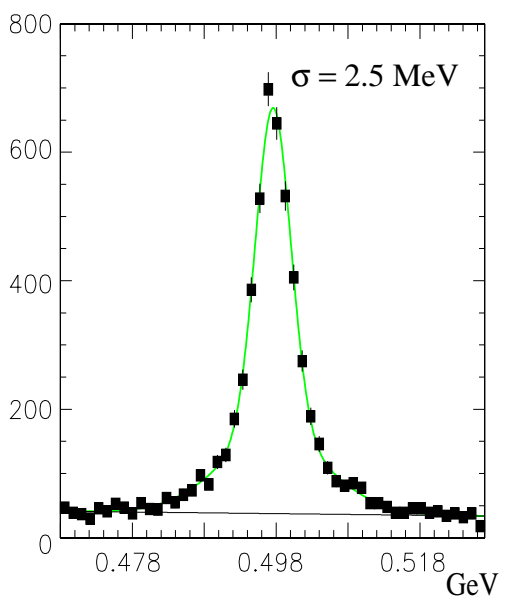

Figure 5: Reconstructed $K^{0}$ signal.

The CDC is composed of 50 anode layers (18 stereo) and 3 cathode layers. It is operated with a $\mathrm{He} /$ ethane 50/50 mixture. The design positional resolution is $\sigma_{R \phi}=130 \mu \mathrm{m}$ and $\sigma_{z}=200-1000 \mu m$, and the $\mathrm{dE} / \mathrm{dx}$ resolution is $6 \%$. Extensive CDC calibration has been done using cosmic ray samples. The performance is quite close to the design value (figure $\overline{4_{1}^{\prime}}$ ). The $K_{S}^{0}$ mass distribution demonstrates the overall 
tracking performance. By cutting on vertex quality and impact parameter, we obtain a clean $K_{S}^{0}$ sample (figure

\section{Particle identification}

Particle identification is the most critical key point for the detector for B-physics. We use $\mathrm{dE} / \mathrm{dx}$ in $\mathrm{CDC}$, TOF and ACC as particle identifying devices.

\section{Bhabha events}

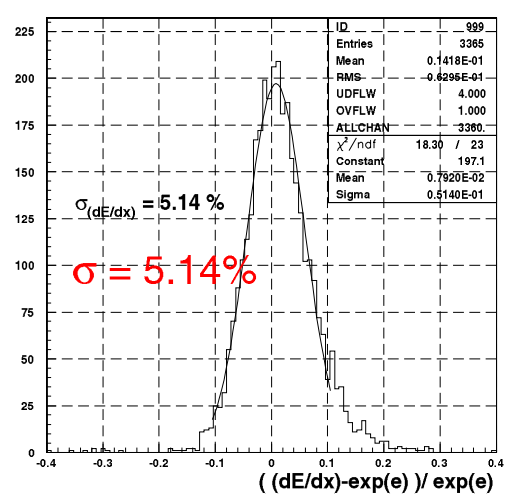

Figure 6: $\mathrm{dE} / \mathrm{dx}$ distribution for Bhabha events.

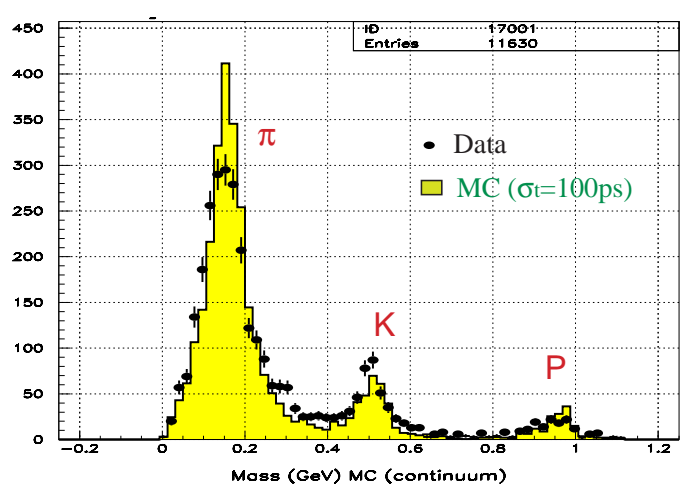

Figure 7: Mass distribution of charged tracks given by TOF (dots). Here Monte Carlo (histogram) assumes the time resolution of $100 \mathrm{ps}$.

Figure $\overline{6} \overline{6}_{1}^{4}$ shows the $\mathrm{dE} / \mathrm{dx}$ of Bhabha event sample with $80 \%$ truncated mean which gives resolution as is expected.

The TOF counter system is composed of 128 modules of plastic scintillator, read out by fine mesh photomultipliers which are developed to operate in a magnetic field. The design time resolution is $\sigma_{t}=100 \mathrm{ps}$. Early data showed that the time resolution for Bhabha events was 125 ps. Figure $\overline{\overline{7}_{1}}$, shows clear separation of the charged particle mass for hadronic tracks with $p<1.2$ $\mathrm{GeV} / \mathrm{c}$.

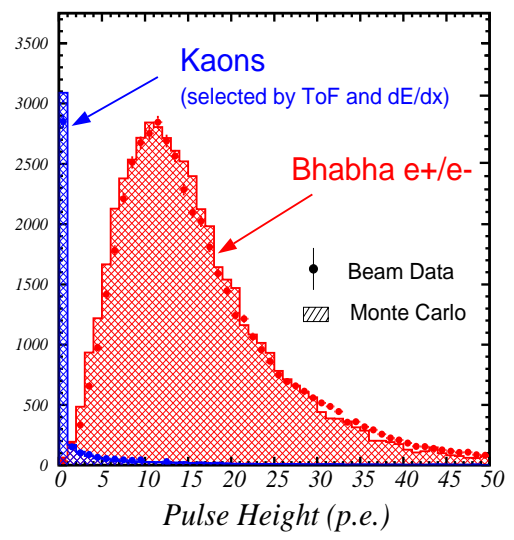

Figure 8: Pulse height distrinution of kaons and electrons selected by TOF and $\mathrm{dE} / \mathrm{dx}$.

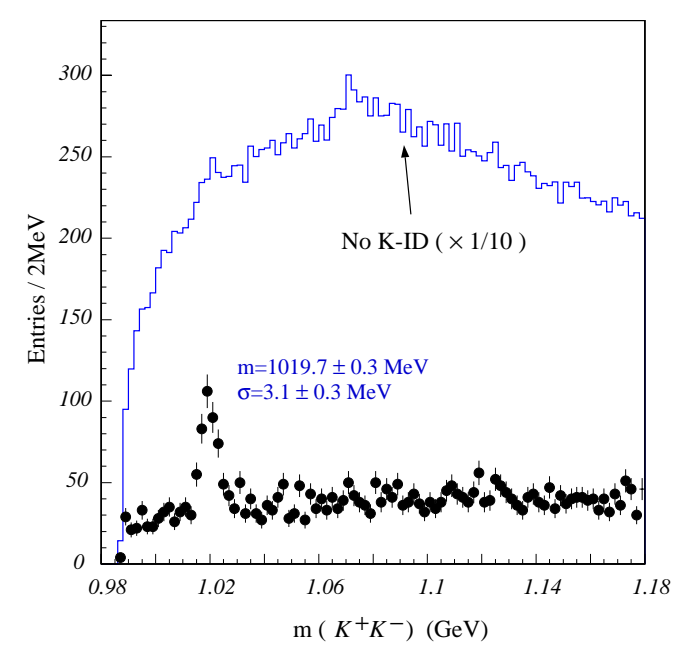

Figure 9: $\phi \rightarrow K^{+} K^{-}$signal w/o (histogram) and with K-ID (dot). Note that the normalization of the histogram without $\mathrm{K}$-id is reduced by a factor of ten.

Higher momentum particles are identified by Silica Aerogel Cherenkov Counter (ACC) with refractive index of 1.01 - 1.03. The ACC has 960 blocks of aerogel radiator in barrel region and 228 blocks in the endcap region. Cherenkov photons are detected by one or two fine mesh photomultiplier(s). Figure $\overline{1}$ shows the typical example of ACC pulse height distribution for kaons and electrons. 


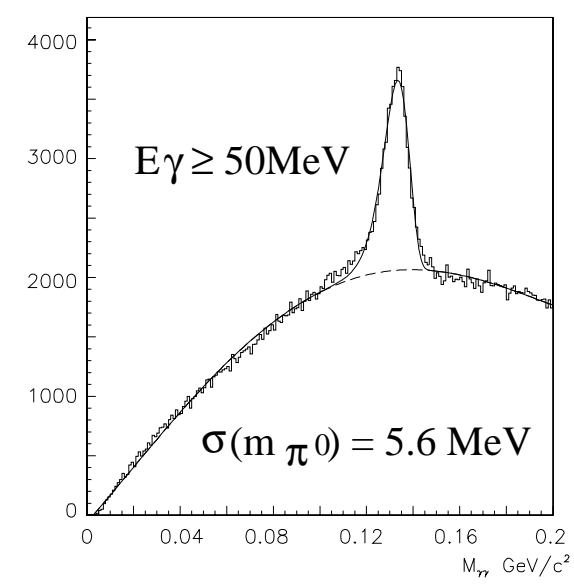

Figure 10: $\pi^{0}$ signal in ECL with $E_{\gamma} \geq 50 \mathrm{MeV}$.

$K / \pi$ identification combines all the PID devices. Figure' 9 'shows an example of how it works. Requiring that the kaon probability be larger than 0.8 for both tracks, a clear $\phi$ signal can be extracted from a large combinatorial background. An inclusive $D^{0}$ signal is also clearly seen with $\mathrm{K}$-id. Again without PID no clear peak is visible.

\section{Photon detection and electron ID}

The electromagnetic calorimeter (ECL) of BELLE consists of $\sim 9000$ fine segmented $\mathrm{CsI}(\mathrm{Tl})$ crystals, each $30 \mathrm{~cm}$ long. The designed energy resolution is $\sigma_{E} / E=0.67 \% / \sqrt{E} \oplus 1.8 \%$ ( $E$ in $\left.\mathrm{GeV}\right)$. Calibration of the calorimeter has been performed using cosmic rays and Bhabha events. We can see a clean $\pi^{0}$ signal with a cut of $E_{\gamma} \geq 50 \mathrm{MeV}$ (figure $\overline{1}^{1} \overline{0}_{-}^{\prime}$ ).

Making use of shower shape in the ECL, the matching of the ECL clusters and charged tracks, $\mathrm{dE} / \mathrm{dx}$ in the CDC and pulse height in the ACC, electron signals can be picked out of the hadronic event sample. Figure 1.1. presents the effect of the electron identification mentioned above, showing a nice peak around 1.0 in the E/p plot.

\section{The $\Upsilon(4 S)$ scan}

The most important physics issue in the commissioning stage is whether we are really getting $B$ mesons, and find out the optimum energy point to be a "B-factory". A preliminary study of the $R_{2}$ distribution of hadronic event sample, taken

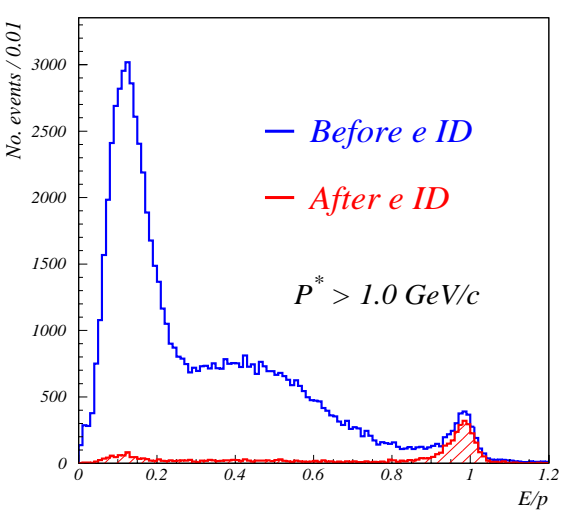

Figure 11: E/p distribution before and after $e^{ \pm}$ identification (hatched).

at the energy at which we started beam collisions, showed that we have about $23 \% B \bar{B}$ contribution over continuum. More direct confirmation is obviously to find the peak of $\Upsilon(4 S)$. The energy scan was carried out before we started the luminosity runs. Figure $12_{1}^{\prime}$ shows the energy dependence of the number of events with $R_{2}<0.2$ normalized by barrel Bhabha events. A cut in $R_{2}$ was placed to enhance the $B \bar{B}$ contribution. The distribution showed that the central value was $10.584 \mathrm{GeV}$ in the KEKB energy scale. The absolute value of the peak energy is yet to be determined. The above result was cross checked by different methods such as measuring muon spectrum, $e^{-}\left(e^{+}\right)$spectrum and $\pi^{0}$ yield. All the results were consistent.

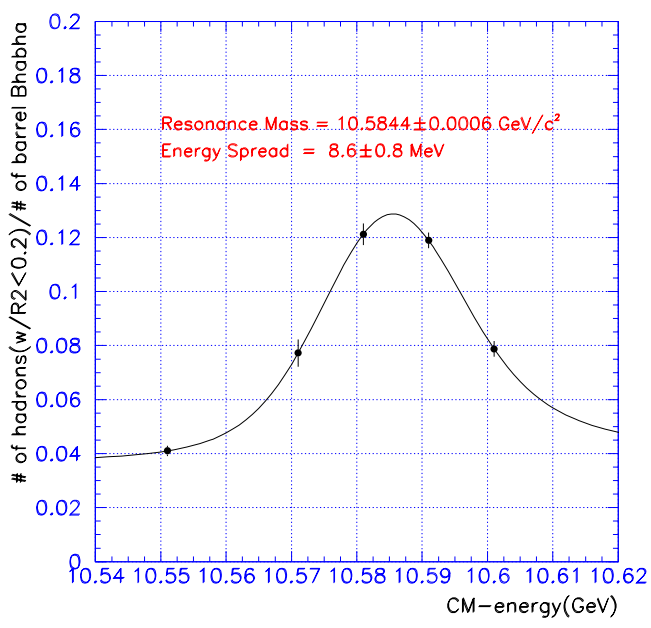

Figure 12: Energy scan for $\Upsilon(4 S)$. 


\section{Beam background problems}

Beam background in the detector is the most critial issue for high current collider like KEKB. The present background in BELLE is marginal, and improvement is needed towards higher luminosity runs. At present, background from the LER is under control and does not have serious problems. However, the HER gives high background in all detectors. The background shows two or more components, one is peaked in the forward region, and the other is widely distributed. Spent electrons and syncrhotron radiaton are suspected sources.

These backgrounds cause problems which are most severe for the CDC and SVD. These include inefficiency, frequent $\mathrm{HV}$ trips of the CDC which impede data taking and the potential danger of ageing. In late July, a large drop in the gain of the VA1 readout chips of the innermost SVD layer was observed. This is believed to be caused by synchrotron radiation with a critical energy of a few $\mathrm{KeV}$, which was unfortunately not expected in the simulation and somehow not checked by monitoring devices. We are going to replace the damaged SVD with a new set of silicon detector, SVD 1.1, with much better synchrotron radiation shielding. We also suffered from synchrotron radiation with energy in the 30 - $50 \mathrm{KeV}$ range. This background is presumably generated at the super conducting quadrupole and back scattered from a section of the downstream vacuum chamber made of alminium. This will be reduced by replacing part of the vacuum chamber with a copper coated one.

\section{Summary}

BELLE/KEKB have started beam collision runs. The full BELLE system is working, and progress is being made in detector calibration. The luminosity exceeded $10^{32} \mathrm{~cm}^{-2} \mathrm{~s}^{-1}$ and the energy scan was completed. On the $\Upsilon(4 S)$ peak we accumulated an integrated luminosity of $\sim 20 p b^{-1}$ by the end of July. Physics analysis are on going using the data sample.

During the summer shutdown, we added RF cavities in order to be able to store higher beam currents. We also replaced the SVD with SVD
1.1 , and replaced a section of the vacuum chamber with a copper coated one. At the same time, we reinforce the background shielding in all possible place to reduce beam background.

In the fall of 1999 we will be running untill the end of the year, aiming to collect $\sim f b^{-1}$ with an averaged luminosity of $10^{33} \mathrm{~cm}^{-2} \mathrm{~s}^{-1}$. In the year 2000, we hope to have a luminosity of a few times $10^{33} \mathrm{~cm}^{-2} \mathrm{~s}^{-1}$, and accumulate an integrated luminosity of $\sim 10 \mathrm{fb}^{-1}$ by summer.

$\mathrm{KEKB} / \mathrm{BELLE}$ is making progress and soon will be a major contributer to the B-physics community.

\section{References}

[1] BELLE collaboration; Academia Sinica, Aomori University, Buker Institute of Nuclear Phyics, Chiba University, Chuo University, University of Cincinatti, Institute of Cosmic Ray Reseaches(University of Tokyo), Fukui University, GyeonSang National University, University of Hawaii, Institute of High Energy Physics, Joint Crystal Collaboration Group, Kanagawa University, KEK, Korea University, Krakow Institute of Nuclear Physics, Kyoto University, Melbourne University, Mindanao State University, Nagasaki Insitute of Applied Science, Nagoya University, Nara Women's University, National Central University, National Kaoshin Normal University, National Lien Ho C.T.C., National Taiwan University, Nihon Dental College, Niigata University, Osaka University, Osaka City University, Princeton University, Saga University, SankyunKwan University, University of Science and Technology of China, Seoul National University, Institue of Single Crystal, Sugiyama Jogakuen University, University of Sydney, Institute of Theoretical and Experimental Physics, Toho Univeristy, Tohoku University, Tohoku-Gakuin University, University of Tokyo, Tokyo Institute of Technology, Tokyo Metropolitan University, Tokyo University of Agricalture and Technology, Toyama National College of Maritime Technology, University of Tsukuba, Utkal University, Yonsei University, and Virgina Polytechnic Institute

[2] BELLE Technical Design Report; KEK Report 95-1 